\title{
Review of Mechanisms of Nanograin Generation
}

\author{
N.V.Skiba ${ }^{1,2}$ \\ ${ }^{1}$ Institute of Problems of Mechanical Engineering, Russian Academy of Sciences, Bolshoy 61 VO, \\ St. Petersburg 199178, Russia \\ ${ }^{2}$ Peter the Great St. Petersburg State Polytechnic University, Politechnicheskaya 29,St. Petersburg 195251, Russia
}

Received: November 25, 2019

\begin{abstract}
A brief review of the theoretical models which describe specific plastic deformation mechanisms of nanograin generation in nanocrystalline and ultrafine-grained materials is presented. In the framework of the models, formation of new nanograins and nanograin chains occur through stress-driven splitting and migration of grain boundaries near crack tips and grain boundary structures containing disclination dipoles and quadrupoles. Stress-driven migration of mobile grain boundaries serves as a specific plastic deformation mode and results in formation of new nanograins and nanograin chains.
\end{abstract}

\section{INTRODUCTION}

Ultrafine-grained (UFG) and nanocrystalline materials (NC) characterized by superior strength represent the subject of rapidly growing research efforts motivated by a wide range of their current and potential applications; see, e.g., [1-4]. The UFG structure in bulk metallic materials is typically formed due to severe plastic deformation that causes grain refinement in initially coarsegrained structures. In order to control the final UFG structure in severely deformed materials, it is highly interesting to understand and describe both the nature and micromechanisms for deformation-induced grain refinement. Despite of the progress in this area $[5,6]$, micromechanisms for deformation-induced grain refinement are still the subject of intense debates. In these circumstances, of particular interest is the stress-driven grain boundary migration which by definition represents a plastic deformation mode carried by migrating grain boundaries $[7,8]$. It is conventionally treated that the stress-driven grain boundary migration carries both nanoscale plastic flow and grain growth $[7,8]$. In particular, this role of the stress-driven grain boundary migration is indirectly supported by experimental observations $[9,10]$ of the athermal grain growth in the vicinities of cracks in NC materials. Recently, it has been suggested that the stress-driven grain boundary migration plays an alternative role as a process carrying nucleation of new grains in NC and UFG materials [11-14]. In doing so, the grain nucleation is considered as a process occurring through splitting and migration of grain boundaries, in parallel with splitting and movement of grain boundary disclinations (rotational defects) [1114]. In papers [11-14], the two types of the initial grain boundary structure (before the grain nucleation) were considered: a highly stressed, disclination-free region near a crack tip of a NC solid [13], and a grain boundary structure containing a disclination dipole in the vicinity of a triple junction of grain boundaries $[11,12,14]$. Whereas, in paper [15], rather similar situation of nanograin chain formation was considered. In this situation, the initial grain boundary structure contains a quadrupole of disclinations located at grain boundary junctions of a previously formed nanograin in a $\mathrm{NC}$ or UFG specimen. The final structure contains two neighboring nanograins which are elongated along the same direction and may serve as nuclei for experimentally observed [16-18] nanograin agglomerates (groups of nanograins with low-angle and/or special boundaries within comparatively large grains having high-angle boundaries) in NC and UFG materials.

Corresponding author: N.V. Skiba, e-mail: nikolay.skiba@ gmail.com 


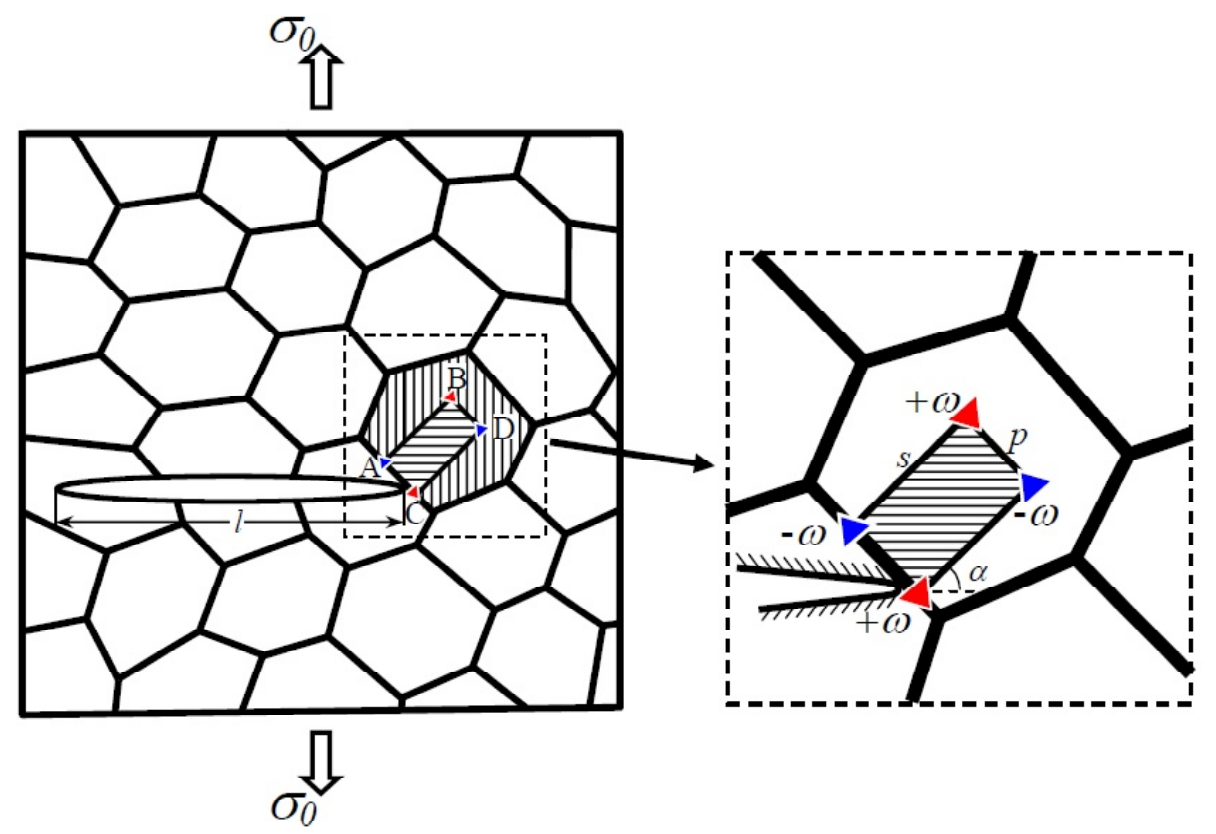

Fig. 1. Generation of a new nanograin and associated disclination quadrupole near a crack tip in deformed NC material.

\section{FORMATION OF NANOGRAINS NEAR CRACK TIPS}

Paper [13] was concerned with nanograin nucleation in disclination-free region near crack tip. In the paper [13], a NC specimen with the mean grain size $d$ contains a mode I crack and is subjected to one-axis tensile load $\sigma_{0}$. Fig. 1 schematically shows a two-dimensional section of an NC solid with a flat crack of length $l$. Following the approach [11], the nanograin nucleation can serve as a special deformation mechanism occurring through splitting of a pre-existent GB $A C$ into immobile boundary $A C$ and mobile boundary $B D$, migration of GB $B D$, and formation of two new grain boundaries $A B$ and $C D$ (Fig. 1). The nucleation of the nanograin $A B C D$ is accompanied by formation of a quadrupole $A B C D$ of wedge disclinations with the strengths $\pm \omega$ near the crack tip [11] (Fig. 1). The new nanograin has a rectangular form with characteristic sizes $s$ and $p$ (serving also as distances between the disclinations located at junctions of the nanograin), and its orientation relative to the crack plane is characterized by angle $\alpha$ (Fig. 1).

The nanograin nucleation (Fig. 1) is characterized by the energy difference $\Delta W=W_{2}-W_{1}$, where $W_{1}$ is the energy of the solid in its initial state containing the crack, and $W_{2}$ is the energy of the solid in its final state containing both the crack and new nanograin. The nanograin nucleation is energetically favorable, if $\Delta W<0$. The energy difference $\Delta W$ (per unit length along the axis $z$ ) can be represented as the sum of the three terms [13]:

$$
\Delta W=E^{q}+E^{q-\sigma}+E_{g b},
$$

where $E^{q}$ is the proper energy of the disclination quadrupole, $E^{q-\sigma}$ is the energy that characterizes the interaction between the disclination quadrupole and the shear stress field $\sigma_{i j}^{c}$ created by the external tensile load $\sigma_{0}$ in the solid containing the crack, and $E_{g b}$ is the sum energy of the three new grain boundaries $A B, C D$, and $B D$.

In a first approximation (neglecting the effects of the crack on the disclination quadrupole stresses), the proper energy $W^{q}$ of the disclination quadrupole is given by the following standard expression [19] for the disclination quadrupole energy in an infinite, elastically isotropic solid [13]:

$$
E^{q}=\frac{D \omega^{2} p^{2}}{2}\left(\left(1+t^{2}\right) \ln \left[1+t^{2}\right]-t^{2} \ln t^{2}\right),
$$

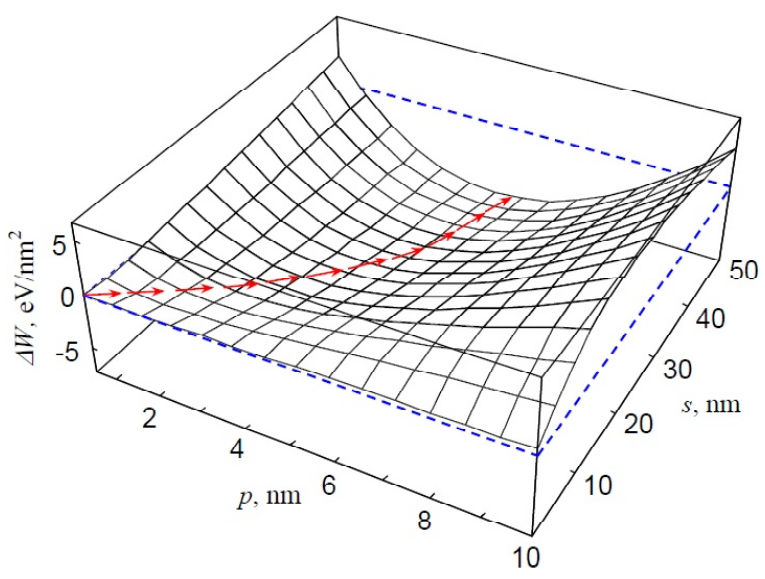

Fig. 2. Dependence of the energy difference $\Delta W$ on the nanograin sizes $s$ and $p$. 
(a)

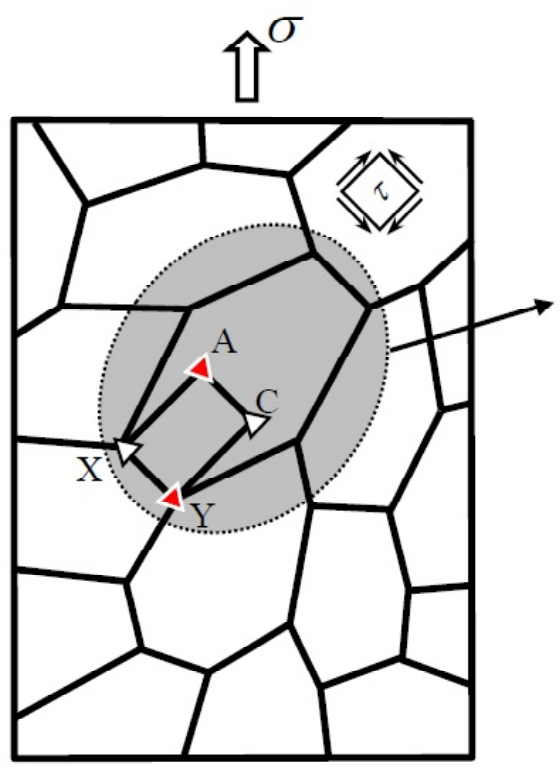

(b)
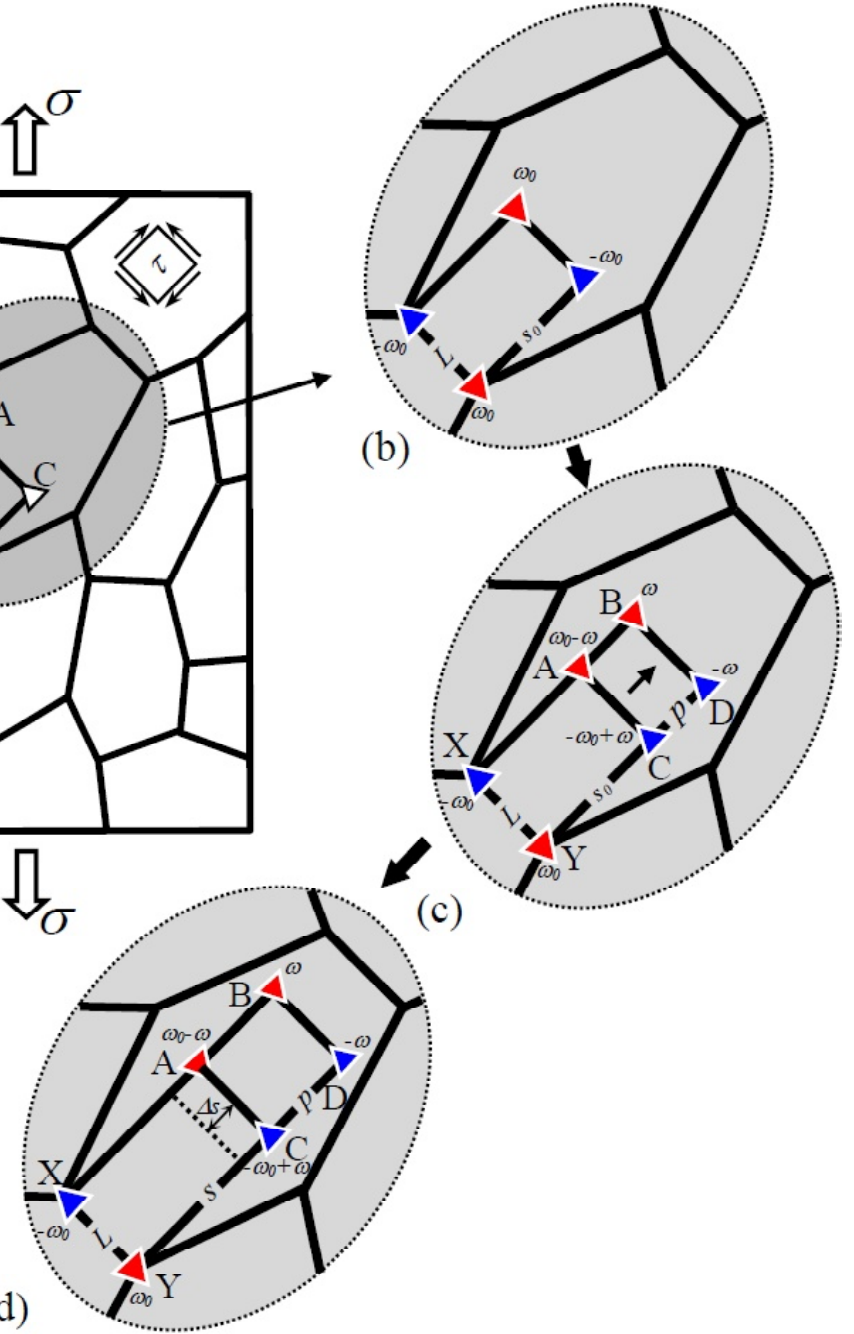

Fig. 3. Formation of a nanograin chain near disclinated nanograin in an UFG specimen. (a) A mechanically loaded specimen with the UFG structure. (b) Initial state with previously formed nanograin $A C Y X$ containing disclination quadrupole. (c) Formation a new nanograin $A B D C$ through stress-driven splitting and migration of the grain boundary $A C$. (d) Formation of a nanograin chain due to growth (elongation) of grains $A C Y X$ and $A B D C$.

where $t=s / p$.

The energy $E^{q-\sigma}$ figuring in formula (2) is written as follows [13,20]:

$$
E^{q-\sigma}=-\omega \int_{S} \sigma_{x y}^{c} d S^{\prime},
$$

where $S$ is the area bounded by the disclination quadrupole, $S^{\prime}$ is the integration parameter, and $\sigma_{x y}^{c}$ is a component of the stress field $\sigma_{i j}^{c}$ (in the coordinate system (x,y); see Fig. 1) created by the external tensile load $\sigma_{0}$ in the solid containing the crack. The expression for $\sigma_{x y}^{c}$ can be found in Ref. [21]. The energy $E_{g b}$ of the new grain boundaries $A B, C D$, and $B D$ is evidently given by formula [13]:

$$
E_{g b}=\gamma_{b}(2 s+p),
$$

with $\gamma_{b}$ being the grain boundary energy per unit area of a boundary plane.
Formulas (1)-(4) allow one to find the energy difference $\Delta W[13]$. The dependence of the energy difference $\Delta W$ on the sizes $s$ and $p$ of the new nanograin in the example case of $\mathrm{NC}$ ceramic $\alpha-\mathrm{Al}_{2} \mathrm{O}_{3}$ is presented in Fig. 2. In calculations, the following typical values of parameters of $\alpha-\mathrm{Al}_{2} \mathrm{O}_{3}$ were used [22,23]: $G=169 \mathrm{GPa}, \nu=0.23$, $\gamma=1.5 \mathrm{~J} / \mathrm{m}^{2}, \gamma_{b}=0.5 \mathrm{~J} / \mathrm{m}^{2}$. The external stress $\sigma_{0}$ is supposed to be equal to $1 \mathrm{GPa}$; this value is typical for mechanical tests of nanocrystalline ceramics. The crack length $L$ is chosen as $L=800 \mathrm{~nm}$. This value is close to (but slightly lower than) the Grifith crack length in $\alpha-\mathrm{Al}_{2} \mathrm{O}_{3}$ at the considered stress level. The characteristic angle is chosen as $\alpha=70^{\circ}$.

Arrows in Fig. 2 show evolution in the space $(s, p)$, corresponding to the most energetically favorable formation/growth of the new nanograin. As it follows from Fig. 2, the most energetically favorable growth of the new nanograin is specified by a simultaneous increase 
in the nanograin sizes $s$ and $p$. That is, for any value of the size $s$, the equilibrium rectangular shape (corresponding to the minimum energy difference $\Delta W(s, p)$ of the new nanograin is specified by equilibrium size $p_{e q}$.

Thus, the nanograin generation through stressdriven splitting and migration of grain boundaries near crack tips is energetically favorable in wide ranges of parameters of deformed NC materials. Formation of new nanoscale grains and associated disclination quadrupoles near crack tips relaxes stresses concentrated near these tips and enhances the fracture toughness in NC materials.

\section{FORMATION OF NANOGRAIN CHAINS NEAR DISCLINATED NANOGRAINS}

In paper [15], a model situation of nanograin chains formation in the vicinities of previously nucleated nanograins containing disclination quadrupoles is considered (Figs. 3a and 3b). In the framework of the model [15], a new nanograin is nucleated in the vicinity of the pre-existent nanograin as follows. The grain boundary $A C$ splits into the immobile grain boundary (also called $A C$ ) and the mobile grain boundary $B D$ that migrates under the external shear stress $\tau$ (Fig. 3c). The migration causes plastic flow (within the area swept by the migrating boundary $B D$ ) and is accompanied by formation of new grain boundaries $A B$ and $C D$ (Fig. 3c). The junction points $B$ and $D$ of the mobile grain boundary contain the disclinations with strengths $\pm \omega$. These disclinations form a mobile dipole configuration and are resulted from splitting of the initial disclinations $A$ and $C$ with strengths $\pm \omega_{0}$ (Figs. $3 \mathrm{~b}$ and $3 \mathrm{c}$ ). As a result of the splitting transformations of the grain boundary $A C$ and the disclinations, a new disclinated nanograin $A B C D$ is formed (Fig. $3 \mathrm{c}$ ).

The distance between the grain boundaries $A C$ and $B D$ is denoted as $p$. In general, during the structural transformation, the external shear stress $\tau$ can cause migration of the grain boundary $A C$ as well (Fig. 3d). In these circumstances, geometry of the structural transformation (Figs. 3b-3d) is specified by changes in both the sizes $p$ and $s$. Finally, note that a series of structural transformations like that shown in Figs. 3b-3d can produce new nanograins. Nanograin chains (Fig. 3d) serve as nuclei of nanograin agglomerates (groups of nanograins with low-angle and/or special boundaries within comparatively large grains having high-angle boundaries) that typically form in NC and UFG materials under severe plastic deformation [16-18].

The splitting transformation (Figs. 3b-3d) is characterized by the energy change $\Delta W=W_{2}-W_{1}$, where $W_{1}$ is the energy of the defect configuration in its initial state with the $\omega_{0}$ - disclination quadrupole (Fig. $3 \mathrm{~b}$ ), and $W_{2}$ is the energy of the defect configuration in its final state (Fig. 3d). The transformation is energetically favorable, if $\Delta W<0$. In terms of the theory of defects in solids, the energy change $\Delta W$ is written as follows [15]:

$$
\begin{aligned}
& \Delta W=E_{s}^{\omega_{0}}+E_{s}^{\omega}+E_{s}^{\omega_{0}-\omega}+E_{\mathrm{int}}^{\omega_{0}-\omega}+E_{\mathrm{int}}^{\omega_{0}-\left(\omega_{0}-\omega\right)}+ \\
& E_{\mathrm{int}}^{\omega-\left(\omega_{0}-\omega\right)}+E_{\gamma}-E_{\tau}-E_{s}^{q} .
\end{aligned}
$$

Here $E_{s}^{\omega_{0}}, E_{s}^{\omega}, E_{s}^{\omega_{0}-\omega}$ and $E_{s}^{q}$ are the proper elastic energies of the dipole $X Y$ of $\pm \omega_{0}$-disclinations, the dipole $B D$ of $\pm \omega$-disclinations, the dipole $A C$ of $\pm\left(\omega_{0}-\omega\right)$-disclinations and the initial quadrupole $X A C Y$ of $\pm \omega_{0}$ - disclinations, respectively; $E_{\text {int }}^{\omega_{0}-\omega}$ is the energy that characterizes the elastic interaction between the dipole $X Y$ of $\pm \omega_{0}$-disclinations and the dipole $B D$ of $\pm \omega$ - disclinations; $E_{\mathrm{int}}^{\omega_{0}-\left(\omega_{0}-\omega\right)}$ is the energy that characterizes the elastic interaction between the dipole $X Y$ of $\pm \omega_{0}$-disclinations and the dipole $A C$ of $\pm\left(\omega_{0}-\omega\right)$-disclinations; $E_{\text {int }}^{\omega-\left(\omega_{0}-\omega\right)}$ is the energy that characterizes the elastic interaction between the dipole $B D$ of $\pm \omega$-disclinations and the dipole $A C$ of $\pm\left(\omega_{0}-\omega\right)$-disclinations; $E_{\gamma}$ denotes the sum of the specific energies (per unit area) of the new grain bounda-
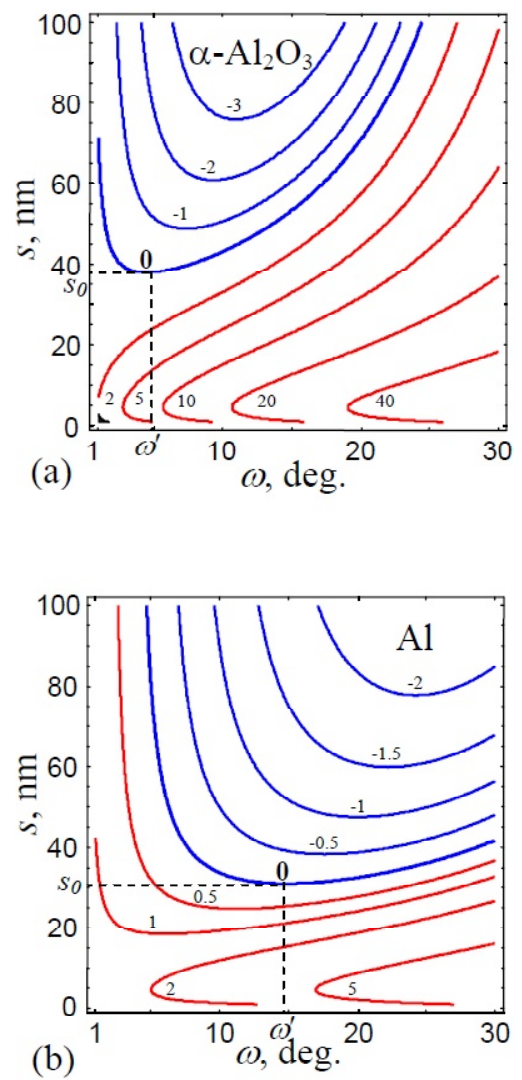

Fig. 4. The map of the energy difference $\Delta W$ in the coordinate plane of $\omega$ and $s$ at the external stress value $\tau=1$ $\mathrm{GPa}$, for (a) NC ceramics $\alpha-\mathrm{Al}_{2} \mathrm{O}_{3}$ (sapphire) and (b) NC $\mathrm{Al}$. 
ries $A B, C D$, and $B D$; and $E_{\tau}$ is the work spent by the external shear stress $\omega$ to displacements of the disclination dipoles $A C$ and $B D$ (Fig. 3d).

After some calculation one can find the expression for the energy change DW (see [15], for details):

$$
\begin{aligned}
& \Delta W=\frac{D \omega^{2}}{2}\left(L^{2} \ln \frac{L^{2}+p^{2}}{L^{2}}+p^{2} \ln \frac{L^{2}+p^{2}}{p^{2}}\right)+\frac{D \omega_{0} \omega}{2}\left(L^{2} \ln \frac{L^{2}}{L^{2}+s^{2}}+L^{2} \ln \frac{L^{2}+(s+p)^{2}}{L^{2}+p^{2}}\right. \\
& \left.+(s+p)^{2} \ln \frac{L^{2}+(s+p)^{2}}{(s+p)^{2}}-s^{2} \ln \frac{L^{2}+s^{2}}{s^{2}}-p^{2} \ln \frac{L^{2}+p^{2}}{p^{2}}\right)-\left(\omega_{0} \Delta s+\omega p\right) L \tau+(2(p+\Delta s)+L) \gamma_{g b} .
\end{aligned}
$$

The maps $\Delta W$ in coordinates of the arm $s$ of the initial $\pm \omega_{0}$-disclination quardrupole and the disclination strength $\omega$ of the mobile $\pm \omega$-disclination dipole in the exemplary cases of $\mathrm{NC}$ ceramics $\alpha-\mathrm{Al}_{2} \mathrm{O}_{3}$ (sapphire) and $\mathrm{NC} \mathrm{Al}$ (aluminum) are presented in Fig. 4. In calculations, the following typical values of parameters were used [23,24]: $G=169 \mathrm{GPa}$ and $v=0.23$, for $\alpha-\mathrm{Al}_{2} \mathrm{O}_{3}$; and $G=26.5 \mathrm{GPa}$ and $v=0.34$, for Al. The values $\gamma_{g b}$ of the energy of special grain boundaries in $\alpha-\mathrm{Al}_{2} \mathrm{O}_{3}$ and $\mathrm{Al}$ are as follows: $\gamma_{g b}^{A L_{3} O_{3}}=0.05 \mathrm{~J} / \mathrm{m}^{2}$ [25] and $\gamma_{g b}^{A l}=0.1 \mathrm{~J} / \mathrm{m}^{2}$ [26]. The arm and strength of the initial $\pm \omega_{0}$-disclination dipole $A C$ (Fig. 1b) are taken as $L=10 \mathrm{~nm}$ and $\omega=30^{\circ}$, respectively.

As it follows from Fig. 4, the splitting process is energetically favorable when the initial distance between the grain boundaries $X Y$ and $A C$ has value of $s_{0} \approx 37 \mathrm{~nm}$ at the disclination strength $\omega^{\prime} \approx 5^{\circ}$, for $\alpha-\mathrm{Al}_{2} \mathrm{O}_{3}$ (Fig. $4 \mathrm{a}$ ); and $s_{0} \approx 31 \mathrm{~nm}$ at $\omega^{\prime} \approx 14^{\circ}$, for $\mathrm{Al}$ (Fig. 4b). In both the cases under examination, when the distance $s$ (with the proviso that $s>s_{0}$ ) increases, so does the interval of disclination strength values $\omega$ at which the splitting process is energetically favorable (Fig. 4).

Thus, the splitting transformations of grain boundaries of previously formed nanograins with disclination quadrupoles at their junctions (Figs. 3b-3d) are energetically favorable and can initiate nucleation of new nanograins in nanocrystalline $\alpha-\mathrm{Al}_{2} \mathrm{O}_{3}$ and $\mathrm{Al}$ in wide ranges of their parameters. Nanograin chains (Fig. 3d) are resulted from these transformations.

\section{CONCLUDING REMARKS}

Thus, formation of the nanograins and nanograin chains is energetically favorable in wide ranges of parameters of deformed UFG and NC materials. It is shown that the generation of nanograins near crack tips causes a partial stress relaxation near cracks. Besides, the stress-driven nanograin formation in NC ceramics is expected to be more effective as a deformation and toughening mechanism, compared to the stress-driven grain growth, because migration of pre-existent grain boundaries in nanoceramics is often suppressed by the difference in the chemical composition between the grain interiors and grain boundaries. Also, it is shown that the nanograin chains may serve as nuclei for experimentally observed [16-18] nanograin agglomerates (groups of nanograins with low-angle and/or special boundaries within comparatively large grains having high-angle boundaries) in NC and UFG materials. The suggested theoretical representations on plastic flow occurring through generation of nanograins at grain boundaries in UFG materials are well consistent with the experimental observation [27] of nanograins generation at grain boundaries in cobalt.

\section{REFERENCES}

[1] A.K. Mukherjee, An examination of the constitutive equation for elevated temperature plasticity, Mater. Sci. Eng. A, 2002, vol. 322, no. 1-2, pp. 1 - 22. https://doi.org/10.1016/S0921-5093(01)01115-7

[2] C.S. Pande and K.P. Cooper, Nanomechanics of Hall-Petch Relationship in Nanocrystalline Materials, Progr. Mater. Sci., 2009, vol. 54, no. 6, pp. 689-706. https://doi.org/10.1016/j.pmatsci.2009.03.008

[3] R.Z. Valiev and T.G. Langdon, The Art and Science of Tailoring Materials by Nanostructuring for Advanced Properties Using SPD Techniques, Adv. Eng. Mater., 2010, vol. 12, no. 8, pp. 677-691. https://doi.org/10.1002/ adem.201000019

[4] C.C. Koch, I.A. Ovid'ko, S. Seal, and S. Veprek, Structural Nanocrystalline Materials: Fundamentals and Applications (Cambridge, Cambridge University Press, 2007). https://doi.org/10.1017/CBO9780511618840

[5] Y. Estrin and A. Vinogradov, Extreme grain refinement by severe plastic deformation: A wealth of challenging science, Acta Mater., 2013, vol. 61, no. 3, pp. 782-817. https://doi.org/10.1016/j.actamat.2012.10.038 
[6] T. Sakai, A. Belyakov, R. Kaibyshev, H. Miura, and J.J. Jonas, Dynamic and post-dynamic recrystallization under hot, cold and severe plastic deformation conditions, Prog. Mater. Sci., 2014, vol. 60, pp. 130-207. https://doi.org/10.1016/ j.pmatsci.2013.09.002

[7] M. Dao, L. Lu, R.J. Asaro, J.T.M. De Hosson, and E. Ma, Toward a quantitative understanding of mechanical behavior of nanocrystalline metals, Acta Mater., 2007, vol. 55, no. 12, pp. 4041-4065. https://doi.org/10.1016/j.actamat.2007.01.038

[8] T.J. Rupert, D.S. Gianola, Y. Gan, and K.J. Hemker, Experimental observations of stress-driven grain boundary migration, Science, 2009, vol. 326, no. 5960, pp.1686-1690. https://doi.org/10.1126/ science. 1178226.

[9] D.S. Gianola, S. Van Petegem, M. Legros, S. Brandstetter, H. Van Swygenhoven, and K.J. Hemker, Stress-assisted discontinuous grain growth and its effect on the deformation behavior of nanocrystalline aluminum thin films, Acta Mater., 2006, vol. 54, pp. 2253-2263. https:// doi.org/10.1016/j.actamat.2006.01.023

[10] S. Cheng, Y. Zhao, Y. Wang, Y. Li, X.-L. Wang, P.K. Liaw and E.J. Lavernia // Phys. Rev. Lett. 104 (2010) 255501. https://doi.org/10.1016/ j.actamat.2006.01.023

[11] S.V. Bobylev and I.A. Ovid'ko, Nanograin nucleation initiated by intergrain sliding and/ or lattice slip in nanomaterials, Appl. Phys. Lett., 2008, vol. 92, article 081914. https://doi.org/ 10.1063/1.2885069.

[12] S.V. Bobylev and I.A. Ovid'ko, Nanograin Nucleation through Splitting and Migration of Grain Boundaries in Deformed Nanomaterials, Rev. Adv. Mater. Sci., 2008, vol. 17, no. ", pp. 7689. http://www.ipme.ru/e-journals/RAMS/ no_11708/bobylev.pdf

[13] I.A. Ovid'ko, N.V. Skiba, and A.K. Mukherjee, Nucleation of nanograins near cracks in nanocrystalline materials, Scripta Mater., 2010, vol. 62, no. 6, pp. 387-390. https://doi.org/10.1016/ j.scriptamat.2009.11.035

[14] S.V. Bobylev, N.F. Morozov, and I.A. Ovid'ko, Cooperative grain boundary sliding and nanograin nucleation process in nanocrystalline, ultrafine-grained, and polycrystalline solids, Phys. Rev. B, 2011, vol. 84, article 094103. https://doi.org/10.1103/ PhysRevB.84.094103

[15] N.F. Morozov, I.A. Ovid'ko, and N.V. Skiba, Stress-driven Formation of Nanograin Chains in Nanocrystalline and Ultrafine-grained
Materials, Rev. Adv. Mater. Sci., 2011, vol. 29, no. 2, pp. 180-186. http://www.ipme.ru/e-journals/ RAMS/no_22911/11_ovidko1.pdf

[16] S. Zghal, M.J. Hytch, J.-P. Chevalier, R. Twesten, F. Wu, and P. Bellon, Electron microscopy nanoscale characterization of ball-milled $\mathrm{Cu}-\mathrm{Ag}$ powders. Part I: Solid solution synthesized by cryo-milling, Acta Mater., 2002, vol. 50, no. 19, pp. 4695-4709. https://doi.org/10.1016/S13596454(02)00285-9

[17] Y. Champion, C. Langlois, S. Guerin-Mailly, P. Langlois, J.-L. Bonnentien, and M. Hytch, Near-Perfect Elastoplasticity in Pure Nanocrystalline Copper, Science, 2003, vol. 300, no. 5617, pp. 310-311. https://doi.org/10.1126/ science. 1081042

[18] X.Z. Liao, Y.H. Zhao, Y.T. Zhu, R.Z. Valiev, and D.V. Gunderov, Grain-size effect on the deformation mechanisms of nanostructured copper processed by high-pressure torsion, J. Appl. Phys., 2004, vol. 96, no. 1, pp. 636-640. https://doi.org/10.1063/1.1757035

[19] M.Yu. Gutkin, K.N. Mikaelyan, A.E. Romanov, and P. Klimanek, Disclination Models of Misorientation Band Generation and Propagation, Phys. Stat. Sol. (a), 2002, vol. 193, no. 1, pp. 35-52. https://doi.org/10.1002/1521396X(200209)193:1<35::AID-PSSA35>3.0.CO;2-Y

[20] A.E. Romanov, V.I. Vladimirov, Disclinations in crystalline solids, In: F.R.N. Nabarro (Ed.), Dislocations in Solids (North Holland, Amsterdam, 1992, Vol. 9, pp.191-402).

[21] M.Yu. Gutkin, I.A. Ovid'ko, and N.V. Skiba Crackstimulated generation of deformation twins in nanocrystalline metals and ceramics, Philos. Mag., 2008, vol. 88, No. 8, p. 1137-1151. https:// doi.org/10.1080/14786430802070813

[22] R.G. Munro, Evaluated Material Properties for a Sintered alpha Alumina, J. Am. Ceram. Soc., 1997, vol. 80, no. 8, pp. 1919-1928. https://doi.org/ 10.1111/j.1151-2916.1997.tb03074.x

[23] C.J. Smithells and E.A. Brands, Metals reference book, London, Butter-worths, 1976. https:// www.elsevier.com/books/metals-reference-book/ smithells/978-0-408-70627-8

[24] J.P. Hirth and J. Lothe, Theory of dislocations, Wiley, New York, 1982.

[25] T. Watanabe, H. Yoshida, T. Saito, T. Yamamoto, Y. Ikuhara, and T. Sakuma, Grain boundary energy and atomic structure in alumina bicrystals, Mater. Sci. Forum, 1999, vol. 304-306, pp. 601-606. https://doi.org/10.4028/ www.scientific.net/msf.304-306.601 
[26] G.C. Hasson and C. Goux, Interfacial energies of tilt boundaries in aluminium. Experimental and theoretical determination, Scripta Metall, 1971, vol. 5, no. 10, pp. 889-894. https://doi.org/10.1016/ 0036-9748(71)90064-0
[27] X. Wu, N. Tao, Y. Hong, G. Liu, B. Xu, J. Lu, and K. Lu, Strain-induced grain refinement of cobalt during surface mechanical attrition treatment, Acta Mater, 2005, vol. 53, no. 3, pp. 681-691. https://doi.org/10.1016/j.actamat.2004.10.021 